\title{
Género y ocupación en la justicia federal
}

\section{Gustavo Fondevila y Alberto Mejía}

\section{Resumen}

En los últimos años, el Poder Judicial de la Federación ha mostrado cierto interés en promover la igualdad de trato y oportunidades entre los géneros y la eliminación de todo tipo de violencia hacia las mujeres. Sin embargo, prácticamente no existen estudios que analicen la situación de los diferentes aspectos de la equidad de género en la administración de justicia y que evalúen el impacto de las políticas en la materia en el sistema judicial. Este ensayo pretende evaluar exploratoriamente el desarrollo de la equidad de género en el Poder Judicial de la Federación a partir de la elaboración de unos indicadores simples que permiten medir el grado de avance real de la mujer en la organización institucional de la justicia federal y las trabas institucionales a dicho avance.

Palabras clave: poder judicial, equidad de género, trabajo formal y doméstico.

\footnotetext{
Abstract

In recent years, the Federal Judicial Branch has shown certain interest in promoting equal treatment and opportunities between genders and eliminating all kind of violence against women. However, there are practically no studies that analyze the situations of the diverse aspects
} 
of gender equity in the administration of justice or that assess the impact of gender policies on the judicial system. The aim of this essay is to evaluate the progress advancement of gender equality in the federal judicial branch through an analysis of simple indicators that measure the degree in which women have made real progress in the institutional organization of the federal courts judiciary and the institutional obstacles that hinder such progress.

Key words: Judicial branch, Gender Equity, Formal and Domestic Work

RECEPCIÓN: 4 DE MARZO DE 2013 / ACEPTACIÓN: 13 DE MARZO DE 2014

${ }^{1}$ Traducción propia.

La natural y apropiada timidez y delicadeza que pertenece al sexo femenino lo vuelve inadecuado para la práctica del derecho. (Morello, 1949) ${ }^{1}$

En los últimos años, han ocurrido cambios sustantivos respecto de la equidad de género en México. El tema de la violencia contra las mujeres ha alcanzado una mayor visibilidad, se han promulgado leyes específicas de protección de las mujeres

${ }^{2}$ Publicada en el Diario Oficial de la Federación el 1 de febrero de 2007. Disponible en http://www.dof.gob.mx/ index.php?year $=2007 \&$ month $=02 \&$ day $=01$ (acceso 01.03.13)
(LGAMVLV, 2007) ${ }^{2}$ y promoción de la equidad, se ha ganado terreno en materia de igualdad salarial (GGGR, 2009) y en el número de mujeres que ocupan puestos claves en el Estado (legisladoras y altos funcionarios) y la iniciativa privada (gerentes). 
Sin embargo, estos cambios positivos se ven empañados por retrocesos como el descenso en el número de mujeres que ocupan cargos ministeriales de 16 a 14\% (GGGR, 2009), con los feminicidios de la ciudad de Juárez, ${ }^{3}$

${ }^{3}$ Alertan por nueva ola de feminicidios en Ciudad Juárez. Disponible en http:/ /www.eluniversal.com.mx/notas/ 547120.html (acceso 28.06.11) los altos niveles de violencia de género y la masiva participación femenina en labores domésticas (96.1\%) frente a los hombres (58.4\%) (INEGI, 2011). Estos ejemplos aislados muestran la disparidad de la situación de la mujer en el país y además, revelan el impacto desigual de las leyes en la materia. El desarrollo legislativo no se ha visto siempre acompañado de esquemas efectivos de implementación y las políticas públicas no han tenido un impacto uniforme. A esta situación se suma la falta de estudios que indiquen el grado de eficacia de los distintos mecanismos del Estado en la promoción de la equidad de género.

Frente a esto, el Poder Judicial de la Federación no ha sido una excepción. Su interés por el tema se refleja en la suscripción del Programa para la igualdad entre mujeres y hombres en el 2007 por el Consejo de la Judicatura Federal (CJF) con la finalidad de promover la igualdad de trato y oportunidades entre los géneros y la eliminación de todo tipo de violencia hacia las mujeres. Sin embargo, prácticamente no existen estudios o programas que analicen la situación de todos los aspectos de la equidad de género en la administración de justicia y que evalúen el impacto de estos programas en el sistema judicial.

Este ensayo pretende evaluar exploratoriamente el desarrollo de la equidad de género en el Poder Judicial de la Federación a 
partir de la elaboración de unos indicadores simples que permiten medir el grado de avance real de la mujer en la organización institucional de la justicia federal y las trabas institucionales a dicho avance.

\section{La equidad de género en términos legales}

${ }^{4}$ http://www.juridicas.unam.mx/infjur/ leg/conshist/pdf/1917.pdf (acceso 24.02.13)

${ }^{5}$ http://www.un.org/es/documents/ udhr/ (acceso 28.02.13)

${ }^{6}$ http://www.insp.mx/transparencia/ XIV/leyes_federales/refcns/pdfsrcs/ 34.pdf (acceso 28.02.13)

\section{Antecedentes}

La Constitución Política de los Estados Unidos Mexicanos de $1917^{4}$ concedió tanto al varón como a la mujer el goce de los derechos humanos, pero restringía el derecho de voto a las mujeres.

- El 10 de diciembre de 1948 el artículo 1 de la Declaración Universal de los Derechos Humanos $^{5}$ establece la igualdad de todo ser humano.

El 17 de octubre de 1953 se reformó el artículo 34 de la carta magna concediendo el derecho al voto de la mujer. ${ }^{6}$

El 31 de diciembre 1974 se reformó el artículo 4 de la norma fundamental para conceder la igualdad del va-

${ }^{7}$ http://info4.juridicas.unam.mx/ijure/ tcfed/9.htm (acceso 26.02.13)

${ }^{8}$ http://www.scjn.gob.mx/Transparencia/Marco_Normativo/Ley\% 20 Genera $\% 20$ Igualdad $\% 20$ MujeresyHombres.pdf (acceso 24.02. 13) rón y la mujer ante la ley. ${ }^{7}$

La Ley General para la Igualdad entre Mujeres y Hombres publicada el 2 de agosto de $2006^{8}$ que promueve el empoderamiento de las mujeres y se guía por los principios de igualdad, no discriminación y equidad. 


\section{Literatura}

Existe una amplia literatura sobre los problemas de la equidad de género en el ámbito laboral, desde las explicaciones históricas de la desigualdad (Amott y Matthaei, 1996; Padavic y Reskin, 2002), las barreras de la "discriminación de segunda generación" (Sturm, 2001) hasta los procesos de diferenciación social de género (Reskin, 1998, West y Zimmerman, 1987). A su vez, el tema de la subordinación de la mujer en el trabajo no es nuevo y ha sido extensamente estudiado con categorías biologicistas (Lorber, 2001), culturales (Freedman, 2002), sociales (Nieva y Gutek, 1981), educativas (Parker y Pederzini, 2000), etc. para entender las diferencias de género, las asignaciones laborales y el rol secundario que ocupan las mujeres en el mercado laboral.

La equidad de género también ha recibido atención desde la economía (Mincer y Polachek, 1974), teoría del rol de género (Nieva y Gutek, 1981), teorías organizacionales (Kanter, 1977), feministas (Feder y Feder Kittay, 2003), etcétera. Particularmente, en materia de derecho hay una infinidad de trabajos que desarrollan diferentes perspectivas sobre la materia. Por ejemplo, la ocupación laboral de las mujeres en el sistema de administración e impartición de justicia (Lorber y Farrell, 1991; Ehrlich Martin y Jurik, 2007), el género y las decisiones judiciales (Allen, 1987; Facio y Fries, 1999), las estrategias feministas de litigio (Howe, 1991), el feminismo y la teoría crítica del derecho (Olsen, 1995), la construcción jurídica de género (Pitch, 2003), el género en las leyes (MacKinnon, 2000), 
el feminismo en el derecho (Smart, 1989), el sexismo jurídico (Sachs y Wilson, 1978), y finalmente la discriminación de la mujer en las penas y sentencias de los jueces (Villanueva Flores, 1999; Motta y Rodríguez, 2001).

En México, hay también numerosos estudios que desarrollan los problemas locales de la inequidad de género en el país (Ariza, 2006), por ejemplo, en los acuerdos comerciales (Espino, 2009), en la legislación previsional (Pautassi, 2002), en el acceso a los servicios de salud (Gómez, 2002), en la violencia y la masculinidad (Liendro, 1998), en las políticas de educación superior (Rodríguez Gómez, 1999), en los partidos políticos (Cerva, 2008) etcétera pero no hay un estudio sistemático del sistema de impartición de justicia en términos de equidad de género como espacio laboral. Esta deficiencia se muestra en la falta de indicadores que permitan registrar el avance de la mujer en puestos considerados habitualmente masculinos (de dirección, magistrados, jueces, etcétera) En este trabajo se proponen algunos indicadores básicos construidos a partir de la escasa información existente en la justicia federal sobre el tema.

\section{Metodología}

Un estudio que sirva para medir y evaluar el nivel de equidad de género alcanzado en el poder judicial de la federación, debe ser abordado desde diferentes instrumentos y concebido a partir de distintas metodologías. Pero una forma rápida de conocer el estado 
de la materia es a partir de la construcción de unos indicadores cuantitativos simples, que permitan medir (de manera no compleja) el avance de la participación de la mujer en la organización institucional de la justicia federal.

En este sentido, los indicadores propuestos para analizar dicha organización del poder judicial son los siguientes:

1. Proporción total de hombres y mujeres. Este indicador busca conocer la proporcionalidad por sexo de las personas que acceden a los escalones más altos de la actividad jurisdiccional.

2. Distribución por sexo en los distintos puestos. Este indicador busca identificar los puestos que ocupan las personas de acuerdo con su sexo.

3. Distribución etaria por sexo. Este indicador se centra en las edades de las personas de acuerdo con su sexo y su puesto, para conocer el tiempo que la persona tarda en llegar a ocupar puestos directivos de acuerdo a su sexo.

\section{Distribución por sexo de los aspirantes (concursos de oposición).}

Este indicador se enfoca en conocer la distribución por sexo de los/ as aspirantes que se presentan a concurso y de aquellos/as que ganan y obtienen la designación.

La información necesaria para la elaboración de estos indicadores proviene de distintas áreas administrativas del propio Consejo de la Judicatura Federal. ${ }^{9}$ 


\section{Hallazgos}

La función jurisdiccional federal se concentra en el Poder Judicial de la Federación. Su ejercicio jurisdiccional pertenece a la Suprema Corte de Justicia, al Tribunal Electoral, a los Tribunales Colegiados y Unitarios de Circuito y a los Juzgados de Distrito. Ahora bien, el órgano encargado de regular la administración, vigilancia, disciplina y carrera judicial del Poder Judicial de la Federación es el Consejo de la Judicatura Federal, la Suprema Corte de Justicia de la Nación está exenta de la vigilancia y control del mismo.

El referido Órgano de control inició su funcionamiento recién en 1995, como consecuencia de la reforma de 31 de diciembre de 1994 a diversos artículos constitucionales y a la Ley Orgánica del Poder Judicial de la Federación de 26 de mayo del mismo año. Dicho Consejo es un órgano del Poder judicial de la Federación con independencia técnica, de gestión y para emitir resoluciones. En términos generales, la organización judicial corresponde a la figura de once ministros, de los cuales diez integran las dos salas de la Suprema Corte y un ministro presidente que no integra sala pero integra el pleno. Los Tribunales Colegiados están integrados por tres magistrados de Circuito de los cuales uno es el presidente del Tribunal, en tanto que los Tribunales Unitarios como su nombre lo indica están integrados por un magistrado de Circuito; mientras que los Juzgados de Distrito están integrados por un juez. Estos titulares son auxiliados en el estudio y substanciación de los asuntos jurídicos por funcionarios denominados secretarios denomina- 
dos respectivamente de Estudio y Cuenta, de Tribunal o de Juzgado. De igual manera, los actuarios son funcionarios judiciales que auxilian en labores específicas, principalmente las notificaciones (la carrera judicial expuesta en forma ascendente inicia con el puesto de actuario, para ir escalando a secretario, a juez de distrito y termina con el de magistrado de circuito; este escenario es el más común pero no el único, pues también existen casos de funcionarios que "saltan" puestos del escalafón, en específico secretarios de Estudio y Cuenta de la Suprema Corte de Justicia de la Nación que son designados magistrados de Circuito sin ocupar el escaño de juez de Distrito). Además, existen funcionarios judiciales denominados oficiales administrativos que pueden ayudar en funciones jurisdiccionales (elaboración de proyectos de sentencias o cualquier documento jurídico que requiera un estudio especializado por un profesional del derecho).

Estos recursos humanos son la fuerza laboral que mantiene en funcionamiento los órganos jurisdiccionales federales.

\section{Acceso a la carrera judicial}

El nombramiento ${ }^{10}$ realizado por los titulares de los órganos jurisdiccionales es la única vía de acceso a la judicatura federal, ya sea en algún puesto de la carrera judicial o en puestos administrativos-jurisdiccionales.

\footnotetext{
${ }^{10}$ Regulado por el artículo 97 párrafo cuarto de la Constitución Política de los Estados Unidos Mexicanos establece que los Ministros, Magistrados y jueces nombrarán y removerán a los respectivos funcionarios y empleados de los Tribunales de Circuito y de los Juzgados de Distrito, conforme a lo que establezca la ley respecto de la carrera judicial. Los requisitos para ocupar el cargo de actuario o Secretario de Estudio y Cuenta, de Tribunal o de Juzgado es ser Licenciado en Derecho y contar con la constancia de aptitud otorgada por el Instituto de la Judicatura Federal. http:/ /www.diputados.gob.mx/LeyesBiblio/ pdf/1.pdf (acceso 28.06.11)
} 
La siguiente tabla 1 muestra de manera general que el número de mujeres que laboran en el Poder Judicial de la Federación es proporcionalmente superior al de los hombres.

En la tabla 1 no se presenta la información de la cantidad de mujeres y hombres que desempeñan los cargos de oficial administrativa/o; actuaria/o; secretaria/o de estudio y cuenta, de tribunal de juzgado. Intuitivamente se puede considerar que dada la mayor cantidad de mujeres que laboran en el Poder Judicial, esta tendencia debe mantenerse constante en cuanto a la proporción superior de mujeres que realizan el tipo de funciones judiciales referidas.

\section{Tabla 1. Distribución de personal del Poder Judicial de la Federación}

\begin{tabular}{|c|c|c|c|c|c|c|c|c|c|}
\hline \multicolumn{10}{|l|}{ Clasificación } \\
\hline por género & \multicolumn{3}{|c|}{2005} & \multicolumn{3}{|c|}{2006} & \multicolumn{3}{|c|}{2007} \\
\hline & Hombre & Mujer & Total & Hombre & Mujer & Total & Hombre & Mujer & Total \\
\hline Órganos jurídicos & 10,78 & 13,374 & 24,15 & 11,304 & 13,823 & 25,127 & 11,711 & 14,162 & 25,873 \\
\hline \multicolumn{10}{|l|}{ Órganos } \\
\hline administrativos & 1,364 & 978 & 2,342 & 1,402 & 999 & 2,401 & 1,426 & 1,004 & 2,430 \\
\hline \multicolumn{10}{|l|}{ Órganos jurídico- } \\
\hline administrativos & 410 & 334 & 744 & 438 & 367 & 805 & 428 & 355 & 783 \\
\hline Total & 12.554 & 14.686 & 27.240 & 13.144 & 15.189 & 28.333 & 13.565 & 15.521 & 29.086 \\
\hline
\end{tabular}

Fuente: Datos del Consejo de la Judicatura Federal (CJF). 


\section{La distribución por género de los titulares} de los órganos jurisdiccionales

Los órganos jurisdiccionales en funciones hasta el 1 de julio de 2010 es la siguiente (véase tabla 2):

Tabla 2. Órganos jurisdiccionales

\begin{tabular}{cccc}
\hline Juzgados de Distrito & $\begin{array}{c}\text { Tribunales Unitario } \\
\text { Colegiados }\end{array}$ & $\begin{array}{c}\text { Tribunales } \\
\text { jurisdiccionales }\end{array}$ & Total órganos \\
\hline 660 & 362 & 212 & 1234 \\
\hline
\end{tabular}

Elaborada con datos de la Secretaría Ejecutiva de Carrera Judicial, Adscripción y Creación de Nuevos Órganos.

En estos órganos jurisdiccionales, la distribución por género de los titulares de Juzgados de Distrito así como la materia de los mismos es la siguiente tabla 3 .

Como se ve en la siguiente tabla, la cantidad de jueces ha aumentado de manera sostenida y significativa de 2004 a 2007. En ese contexto, la cantidad de mujeres de 2006 a 2007 se logró prácticamente duplicar con respecto al año anterior. Los juzgados de distrito de procedimientos penales federales tienen una problemática propia que consiste en la inexistencia de horarios fijos de trabajo derivado de las funciones propias que realizan (declaraciones preparatorias, autos de término constitucional, órdenes de aprehensión, etcétera). Este factor puede ser un indicador relevante a 
Tabla 3. Dotación de jueces en los Juzgados de Distrito

\begin{tabular}{|c|c|c|c|c|c|c|c|c|c|c|c|c|}
\hline \multicolumn{13}{|l|}{ Juzgados de } \\
\hline Distrito & \multicolumn{3}{|c|}{2004} & \multicolumn{3}{|c|}{2005} & \multicolumn{3}{|c|}{2006} & \multicolumn{3}{|c|}{2007} \\
\hline & Hombr & e Mujer & r Total & Hombre & Mujer & r Total & Hombre & Mujer & r Total & Hombre & e Mujer & r Total \\
\hline Administrativo & 14 & 10 & 24 & 15 & 10 & 24 & 13 & 11 & 24 & 191 & 134 & 325 \\
\hline \multicolumn{13}{|l|}{ Amparos y } \\
\hline Juicios Civiles & 3 & 1 & 4 & 3 & 1 & 4 & 4 & 1 & 5 & 31 & 28 & 59 \\
\hline Civil & 15 & 3 & 18 & 15 & 3 & 18 & 15 & 3 & 18 & 128 & 119 & 247 \\
\hline Civil/trabajo & 4 & - & 4 & 4 & - & 4 & - & - & - & 34 & 21 & 55 \\
\hline \multicolumn{13}{|l|}{ Amparo en } \\
\hline materia penal & 7 & 5 & 12 & 7 & 5 & 12 & 9 & 5 & 14 & 75 & 64 & 139 \\
\hline Penal & 14 & - & 14 & 14 & - & 14 & 13 & 1 & 14 & 114 & 55 & 169 \\
\hline \multicolumn{13}{|l|}{ Procesos penales } \\
\hline federales & 23 & 3 & 26 & 22 & 4 & 26 & 19 & 7 & 26 & 160 & 83 & 243 \\
\hline Mixtos & 161 & 47 & 198 & 138 & 46 & 184 & 137 & 50 & 187 & 1.359 & 915 & 2.274 \\
\hline Trabajo & 1 & 4 & 5 & 2 & 3 & 5 & 4 & 2 & 6 & 39 & 33 & 72 \\
\hline Total & 232 & 73 & 305 & 219 & 72 & 291 & 214 & 80 & 294 & 2.131 & 1.453 & 3.584 \\
\hline
\end{tabular}

Fuente: Datos del CJF.

la hora de estudiar las posibilidades de desempeño de las mujeres en tareas que conllevan este tipo de cargas de trabajo.

En lo que respecta a la cantidad de titulares de Tribunales de Circuito es como sigue en la tabla 4.

Se intuye que la tendencia desfavorable entre el número de magistrados hombres en relación con el de mujeres tiene altas po- 
sibilidades de persistir como tendencia a lo largo del tiempo (Carson, 2004).

Tabla 4. Dotación Magistrados de Circuito

\begin{tabular}{lccc}
\hline & 2004 & 2006 & 2008 \\
\hline Tribunales Colegiados & 508 & 540 & 538 \\
\hline Tribunales Unitarios & 64 & 69 & 75 \\
\hline Total de Magistrados & 572 & 609 & 613 \\
\hline
\end{tabular}

Fuente: Datos del Poder Judicial y su Dirección General de Estadística y Planeación Judicial.

\section{Suprema Corte de Justicia de la Nación}

El cargo de ministro no forma parte de la carrera judicial, pues no se accede por medio de concurso de selección, ni es necesario haber sido juez con anterioridad a su designación. La designación de ministros se rige por reglas constitucionales propias (art. 95 de la CPEUM) cuya designación recae en el Poder Ejecutivo y en el Senado de la Nación.

La Suprema Corte de Justicia de la Nación es el máximo Tribunal del país, integrado como ya se dijo por once ministros, de los cuales actualmente solamente dos de sus integrantes son mujeres (véase tabla 4.1). 
Tabla 4.1. Ministros de la Suprema Corte de Justicia de la Nación (2013)

\begin{tabular}{cc}
\hline Hombres & Mujeres \\
\hline 9 & 2 \\
\hline
\end{tabular}

Porcentaje: $18.18 \%$

\section{La edad promedio de jueces y magistrados} del Quinto Circuito en 2004

La edad promedio puede ser un indicador de la facilidad o rapidez en el ascenso a puestos directivos. Comparativamente puede servir para saber cuánto tiempo tarda una persona de acuerdo a su género en alcanzar un puesto determinado.

Tabla 5. La edad promedio de jueces y magistrados del Quinto Circuito en 2004

\begin{tabular}{lcc}
\hline & Mujeres & Hombres \\
\hline Juzgados de Distrito & 40 & 56 \\
\hline Tribunales Unitarios & 44 & 0 \\
\hline Tribunales Colegiados & 51 & 46 \\
\hline
\end{tabular}

Fuente: Datos de la Secretaria Ejecutiva de Administración del Consejo de la Judicatura Federal. ${ }^{11}$ 
Se aprecia que la edad promedio de juezas y magistradas es significativamente mayor que la de jueces y magistrados. Esto puede ser otro indicador relevante para determinar las inequidades de género que impiden estructuralmente que las mujeres alcancen los mismos puestos que los hombres en los mismos tiempos (en términos estadísticamente comparables). En este punto, un análisis más detallado puede descubrir obstáculos de género en la consecución de ascensos así como con la rapidez para obtenerlos.

\section{Formas de acceso a puestos directivos en el Poder Judicial de la Federación}

El nombramiento y adscripción de los magistrados de circuito y de los jueces de distrito es facultad exclusiva del Consejo de la Judicatura Federal ${ }^{12}$ a través de los concursos internos y externos de oposición para la designación de jueces y magistrados.

El concurso de selección de Jueces y Magistrados del Consejo de la Judicatura Federal ${ }^{13}$ es un mecanismo que examina el conocimiento jurídico y la aptitud psicológica de los aspirantes a través de fases que consisten en exámenes sobre conocimientos jurídicos, exámenes de resolución de un caso práctico y la realización de pruebas psicométricas. ${ }^{14}$

La proporción de mujeres que acceden a los puestos de jueces y magistrados sigue siendo menor

\begin{abstract}
${ }^{12}$ Regulado por el artículo 97 constitucional: Artículo 97. Los Magistrados de Circuito y los Jueces de Distrito serán nombrados y adscritos por el Consejo de la Judicatura Federal, con base en criterios objetivos y de acuerdo a los requisitos y procedimientos que establezca la ley. Durarán seis años en el ejercicio de su encargo, al término de los cuales, si fueran ratificados o promovidos a cargos superiores, sólo podrán ser privados de sus puestos en los casos y conforme a los procedimientos que establezca la ley.

${ }^{13}$ Concursos regulados por los acuerdos generales 30/2008, 57/2008 y 51/2008 del Pleno del Consejo de la Judicatura Federal.

${ }^{14}$ De acuerdo con el Consejero César Esquinca «el sistema parece haberse desgastado, en parte porque se descuidaron los sistemas de control, lo que permitió la intervención de más perso-
\end{abstract}


nas de las debidas, la filtración de material de las pruebas y, por ende, resultados inexplicables desde el punto de vista de los méritos de algunos participantes.» Este consejero propone el abandono de este tipo de selección para dar paso a un sistema de aprobación de un curso impartido por el Instituto de la Judicatura Federal.

15 http://www.cjf.gob.mx/secretarias/ secjacno/Carrera\%20Judicial/ concursos.html (acceso 28.06.11) a la de los hombres que llegan a esas plazas. Esta distribución asimétrica de género de aspirantes que se presentan a estos concursos puede ser otro indicador importante para indagar si existe una variable de género (obstáculo) que explique la incidencia de la tendencia.

Las secretarias de Estudio y Cuenta, de Tribunal y de Juzgado que se presentan a este tipo de concursos de oposición se presuponen aptas para desempeñar el papel de jueces, derivado de las funciones judiciales que realizan cotidianamente en los órganos jurisdiccionales donde laboran. Podría ser interesante hacer un análisis sistemático de los motivos que llevan a las funcionarias judiciales a presentarse o no presentarse a los concursos. Así como los motivos por los cuales son consideradas como no aptas en los concursos de oposición.

\section{Gráfico 1.}

\section{Total de magistrados de circuito y jueces de distrito designados $2008-2009$}

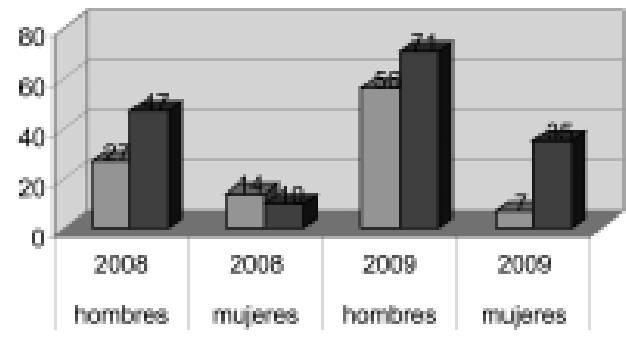

Fuente: Datos del CJF ${ }^{15}$ en el año 2007 no se publicó la lista de ganadores. 


\section{Discusión}

A través de este breve ejercicio de análisis de evidencia empírica se han identificado cuatro áreas que pueden tener importancia en cuanto a la perspectiva de género en el Consejo de la Judicatura Federal:

a) el número de mujeres (15.521: 53.36\%) que labora en el Poder Judicial de la Federación es mayor que el número de hombres (13.565: 46.62\%).

- b) la proporción de mujeres, en términos generales, no se refleja en la proporción de mujeres que ocupan puestos de jueces o magistradas (la mayoría de las mujeres ocupan puestos bajos): 56 magistrados hombres frente a 7 magistradas mujeres en 2009 (una relación de 8 veces);

c) la edad de las mujeres que ocupan puestos de juezas y magistradas es significativamente mayor a la edad de los hombres (a las mujeres les lleva más tiempo llegar a los mismos lugares que los hombres): en los tribunales colegiados la edad promedio de los hombres es de 41 años, frente a 56 años de promedio en las mujeres;

d) la proporción de mujeres que se presentan a los concursos es inferior a la de los hombres (las mujeres no se presentan a los concursos para puestos superiores en la misma proporción que los hombres). 
Estas diferencias sirven para expresar materialmente las inequidades de género persistentes en la distribución y administración de la fuerza laboral. A pesar de las leyes y regulaciones ya mencionadas, el poder judicial federal no ha logrado generar un sistema laboral equitativo. Esto no implica necesariamente ninguna intencionalidad explícita del sistema. En muchas ocasiones, la inequidad de género se debe simplemente a condiciones iguales para ambos géneros. Paradójicamente, la equidad de género se alcanza habitualmente mediante condiciones desiguales (distribución de horarios, de cargas laborales, etcétera) En caso contrario, las políticas organizacionales permiten la segregación por género y esto obliga a las mujeres a enfrentar la exclusión de la cultura del trabajo informal, la hostilidad de la interacción social, acoso sexual, atribuciones laborales diferenciadas y la marginación de mujeres con responsabilidades familiares (Ehrlich Martin y Jurik, 2007: 2). Estos factores terminan provocando el lento avance de las mujeres en posiciones relevantes o su renuncia a ocupar papeles de mayor peso dentro del sistema judicial por el costo secundario que deben asumir (falta de tiempo para la familia, por ejemplo). Sin lugar a dudas, la división de género del trabajo en el sistema de justicia es solamente una parte del proceso de diferenciación social del país, pero el poder judicial tiene una responsabilidad directa en la promoción de la equidad. Los indicadores parecen dejar claro que las leyes son insuficientes para insertar a las mujeres en los altos niveles del poder judicial. Por el contrario es necesario desarrollar políticas públicas orientadas a intervenir directamente en la estructura organizacional del 
sistema de justicia. Los datos señalan que los programas de equidad de género del Consejo de la Judicatura son insuficientes, quizás porque están enfocados en la profesionalización de la justicia en términos individuales sin acompañar este esfuerzo con cambios en la organización y forma del trabajo en los juzgados (Jurik y Musheno, 1986). La profesionalización aumenta los niveles de eficiencia, equidad (en términos generales) y neutralidad de género, pero esto no necesariamente trae aparejado más oportunidades para que las mujeres alcancen roles de importancia en el poder judicial. A pesar de los programas de equidad de género, a las mujeres les sigue costando más esfuerzo, tardan más años y llegan en menor cantidad que los hombres a los mismos puestos.

Estos datos parecen mostrar que las reglas y el funcionamiento de la institución (intencionadamente o no) ha adoptado regulaciones y programas de no discriminación y de equidad de género, pero al mismo tiempo, parecen subsistir prácticas laborales "neutrales" en términos de género, pero que precisamente por esa neutralidad en temas clave del trabajo cotidiano, terminan reduciendo las posibilidades de una equiparación real de hombres y mujeres. Esta discriminación de "segunda generación" incluye desventajas estructurales encubiertas y no conscientes a nivel institucional, muy difíciles de identificar y analizar adecuadamente (Wilkins y Gulati, 1996). Se trata de prácticas que cristalizan formas de la inequidad insertas en la lógica laboral de la institución judicial (Acker, 1990). Se trata de un dispositivo diseñado para hombres al que las mujeres deben adaptarse sin mediaciones: desde los meca- 
nismos de ingreso hasta las calificaciones necesarias para ascender, el aparato laboral de la justicia está diseñado bajo la forma masculina de la competencia, del desempeño, de las calificaciones, del tiempo de trabajo, de los horarios, etcétera. (Morash y Greene, 1986; Cockburn, 1991; Reichman y Sterling, 2002). Esta concepción y estructuración de la labor judicial impide el avance de las mujeres, no de manera formal (precisamente, ya no hay barreras formales explícitas) sino por las características mismas del trabajo en la justicia. Las mujeres con dificultades para adaptarse a esta lógica laboral de género son relegadas a los puestos inferiores del sistema (Carson, 2004), a las tareas consideradas tradicionalmente femeninas (como las funciones secretariales) o de menor status, o bien a las ramas jurídicas también percibidas como "femeninas" (por ejemplo, el derecho de familia: en los juzgados civiles, la relación hombre/mujer en jueces es de 128 a 119 mientras que en los juzgados penales, dicha relación es de 114 a 55) producto de la estratificación judicial. Aunque la institución está en condiciones de repensar y modificar dichas características de tal modo que se logre equiparar los géneros.

\section{Conclusión}

Las dificultades de las mujeres para alcanzar puestos de importancia en la justicia no deben ser analizadas aisladamente sino en el contexto de las condiciones sociales de trabajo de dichas mujeres. La estructura del trabajo organizacional en la sociedad mexicana 
está desbalanceada para la mujer, asignándole responsabilidades desproporcionadas en el cuidado de la familia, tareas domésticas y labores consideradas habitualmente como femeninas. Se las considera como las proveedoras de servicios en el núcleo laboral, lo cual tiene una carga cultural en cuanto al rol general de las mujeres en la familia.

En México el total de hogares censales asciende a 28159 373, de ellos los que reconocen a un varón como el jefe son 21.2 millones y 6.9 los que reconocen a una mujer como tal. En términos relativos de cada 100 hogares censales, 75 son dirigidos por un hombre y 25 por una mujer. Las entidades federativas con las proporciones más altas de hogares dirigidos por una mujer son el Distrito Federal (31.4), Morelos (27.4). ${ }^{16}$

${ }^{16}$ Mujeres y Hombres en México 2011, INEGI, p. 50. http://www.inegi.org.mx/ prod_serv/contenidos/espanol/bvinegi/ productos/integracion/

El clima social de la estructura del trabajo refuerza sociodemografico/mujeresyhombres/ la organización de género de las actividades den2011/MyH2011.pdf (acceso 28.02.13)

tro de las instituciones de justicia y consolida las barreras para la implementación de condiciones laborales neutras en términos de género que dificulta o ralentiza el acceso de mujeres a niveles jerárquicos. A este clima social se suma la cultura de género del trabajo judicial, las llamadas barreras culturales de segunda generación (Ehrlich Martin y Jurik, 2007) que dificultan el avance femenino. Dichas barreras pueden sintetizarse del siguiente modo: 
Disponibilidad de tiempo (Sirianni y Welsh, 1991). Habitualmente, debido a la desigual distribución de la carga familiar en México, las mujeres no disponen de la misma cantidad de tiempo que los hombres para poder dedicarle a la carrera profesional. Esto las afecta particularmente en aquellos puestos que demandan tiempo fuera del horario tradicional de trabajo por la carga misma del servicio (alto número de casos) o bien, porque tienen horarios extendidos (por ejemplo, los juzgados de distrito de procedimientos penales federales) por sus propias funciones.

Concepción masculina del derecho (Rhode, 1988). La ley y su aplicación son consideradas habitualmente como tareas propias del hombre. Por este motivo, la mayoría de las funciones y el ideal mismo del sistema judicial parecen adecuados a la mentalidad masculina, donde las mujeres ocupan un rol secundario o tareas "femeninas" que no tienen un status relevante en el sistema.

- Exclusión de grupos no dominantes (Sturm, 2001). A diferencia de las barreras de primera generación que explícitamente excluyen a las mujeres de ciertas posiciones, las de segunda generación implican prácticas sociales y patrones de interacción (preferencias, expectativas, etcétera) que excluyen y discriminan a los grupos no dominantes (Ehrlich Martin y Jurik, 2007). 
Modelo profesional masculino (Pierce, 1995). La concepción del abogado pero sobre todo del impartidor de justicia está basado en una imagen masculina, con valores considerados socialmente como masculinos: imparcialidad, racionalidad, justicia, etcétera.

Concepción de la mujer (Ehrlich Martin y Jurik, 2007). El ideal de mujer es fundamental para comprender la concepción "judicial" de la misma. En este sentido, los estereotipos de género le asignan un status superior (más trabajador, competente, más comprometido) a los hombres frente a las mujeres.

Falta de mentores femeninos (Reichman y Sterling, 2002). La ausencia de mujeres en posiciones de relevancia dentro del poder judicial también impide la existencia de mentores femeninos que generen redes institucionales que permitan el ascenso de otras mujeres.

Redes sociales informales (Seron y Ferris, 1995). Este punto hace referencia a las redes informales que se generen alrededor del trabajo judicial. La imposibilidad de las mujeres de participar de dichas redes les dificultan el acceso a puestos claves en el sistema, o la asignación de tareas importantes o casos de alto impacto.

Estas "barreras culturales" tienen amplia difusión pero se apoyan inevitablemente en una lógica organizacional neutra al género que tiene impacto negativo en las mujeres. Aunque la modificación de 
la organización del trabajo en el poder judicial no implica necesariamente la eliminación de estas barreras, es posible pensar que las mismas se verían seriamente limitadas o restringidas por la ampliación de oportunidades de las mujeres para alcanzar los niveles más altos del sistema.

\section{Implicaciones de política pública}

Como es posible observar, a pesar de los programas de equidad de género y de los esfuerzos realizados por el poder judicial, no se han alcanzado niveles relevantes de equidad laboral en el sistema. En este sentido, es fundamental elaborar un diagnóstico que permita examinar las desigualdades por razón de género que existen en la actualidad, tanto en el mercado de trabajo (del sistema) como en la política judicial. El análisis de la participación de la mujer en la fuerza de trabajo también debe proporcionar datos de la segregación ocupacional, de las diferencias de retribución y del reparto del tiempo, según el género. También debe servir para abordar el progreso de las mujeres en la función judicial y su acceso a puestos de dirección; obtener estadísticas relacionadas con su avance en los puestos de mayor responsabilidad judicial. Inclusive es importante que proporcione datos sobre la participación masculina y femenina en la educación y la formación judicial. Identificará los obstáculos que impiden o limitan el desarrollo profesional de las mujeres en el ámbito judicial. Asimismo, se deben examinar los motivos que llevan a separar los caminos profesionales de hombres 
y mujeres (horarios, responsabilidades familiares, políticas de selección, etcétera) Y sobre todo, es prioritario obtener información cualitativa clave de los operadores del sistema para conocer la percepción de las mujeres, sus opiniones respecto del funcionamiento del sistema en términos de equidad de género, y que permita conocer las motivaciones de los agentes (mujeres) para no competir en los concursos, o los obstáculos que encuentran en sus carreras para ascender, etcétera.

Independientemente de ese estudio, a partir del presente ensayo se puede inferir que la función judicial requiere de metas de corto y mediano plazo para generar las condiciones de equidad que posibiliten aumentar la proporción de mujeres que participen en los concursos de oposición, y establecer una cuota de género (considerando las aptitudes requeridas en cuanto a la capacidad y aptitud exigida a los juzgadores $)^{17}$ en los resultados de los concursos de oposición. También es necesario generar una propuesta de un nuevo sistema de selección en base a las necesidades particulares de la función judicial y la perspectiva de género.

\begin{abstract}
${ }^{17}$ La selección de los jueces y magistrados presenta problemas derivados de la especialización en los requisitos de la función judicial, la cual debe de estar basada primordialmente en los principios de excelencia y honradez pero es posible considerar la perspectiva de género, cuanto más si se acepta que las funcionarias judiciales elegibles para ocupar los cargos de juez o magistrado demuestran sus aptitudes y capacidades jurídicas día a día en los órganos jurisdiccionales en los cuales realizan en la práctica funciones de auténticos jueces auxiliares derivado de la organización del sistema de impartición de justicia en las ponencias, los juzgados y tribunales.
\end{abstract}

Este trabajo permitirá sentar las bases para remover los obstáculos materiales para alcanzar la equidad real de género. 


\section{Bibliografía}

ACKeR, J. "Hierarchies, Jobs And Bodies: a Theory of Gender Organization”. Gender \&ु Society, 4, 139-158, 1990.

Allen, Hilary. Justice Unbalanced: Gender, Psychiatry, and Judicial Decisions. Milton Keynes: Open University Press, 1987.

Amott, T. y J. Matthaer. Race, Gender And Work: a Multi-Cultural Economic History of Women in United States. Boston, South End Press, 1996.

ARIZA, Marina. "Mercados de trabajo urbanos y desigualdad de género en México a principios del siglo XXI", en Enrique de la Garza y Carlos Salas (coord.) La situación del trabajo en México, 2006, Universidad Autónoma Metropolitana-Instituto de Estudios del Trabajo/Centro Americano para la Solidaridad Sindical Internacional-AFL-CIO/ Plaza y Valdés, 377-411, 2006.

CARson, C. The Lawyer Statistical Report: The U.S. Legal Profession in 2000.

Chicago, Chicago American Bar Foundation, 2004.

CERVA, D. Los partidos políticos frente la equidad de género en México. Estocolmo, International Idea, 2008.

Cockburn, C. In The Way of Women: Men's Resistance to Sex Equality in Organizations. Ithaca, NY, ILR Press, 1991.

EHrLICH Martin, Susan y Nancy JuRIK. Doing Justice, Doing Gender. Thousand Oaks, CA, Sage, 2007.

EsPINO, A. "El impacto de las medidas anticrisis en Centroamérica. Posibles impactos en el empleo de las mujeres y en la conciliación entre el trabajo en el hogar y en el mercado". Foro: Respondiendo a la crisis con equidad de genero. 10-11 de nov., San José de Costa Rica, 2009. 
FACIO, Alda y Lorena Fries (eds.) Género y Derecho. Santiago, La Morada/ Lom/American University, 1999.

Feder, Ellen K, y Eva Feder Kittay (eds.) The Subject of Care: Feminist Perspectives on Dependency. Lanham, Rowman, 2003.

FreEDMAn, E. No turning back; The History of Feminism and the Future of Women. New York, Ballantine Books, 2002.

GGGR. The Global Gender Gap Report. World Economic Forum. Disponible en https://members.weforum.org/pdf/gendergap/report2009.pdf (acceso 27.06.11) 2009.

Goffman, Erving. Stigmate: les usages sociaux des handicaps. Paris, Minuit, 1975.

GÓMEZ, E. "Género, equidad y acceso a los servicios de salud: una aproximación empírica". Revista Panamericana de Salud Publica, 11, 5-6, 2002.

Howe, Adrian. "The Problem of Privatized Injuries: Feminist Strategies for Litigation", en M. Fiman y N. Thomadsen (eds.); At the Boundaries of Law. London, Routledge, 1991.

Instituto Nacional de Estadística y Geografía (INEGI). Disponible en http://www.inegi.org.mx/ (acceso 28.06.11)

Jurik, N. y M. Musheno. "The Internal Crisis of Corrections: Professionalization and the Work Environment". Justice Quarterly, 3, 457480, 1986.

Kanter, R. Men and Women of the Corporation. New York, Basic, 1977.

LEY GENERAL DE ACCESO DE LAS MUJERES A UNA VIDA LIBRE DE VIOLENCIA (LGAMVLV). Disponible en http://www.diputados.gob.mx/LeyesBiblio/ pdf/LGAMVLV.pdf (acceso 27.06.11) 
LIENDRO, E. "Masculinidades y violencia desde un programa de acción en México”, en Valdés, T. y Olavarría, J. (eds.), Masculinidades y equidad de género en América Latina. Santiago, Chile, FLACso-Chile, 1998.

LORBER, J. Gender Inequality: Feminist Theories and Politics. Los Angeles, Roxbury, 2001.

— y Farrell, S. (eds.) The Social Construction of Gender. London, Sage. 1991.

Mackinnon, Catharine A. Women's Lives, Men's Laws. Cam. Mass, Harvard University Press, 2000.

MINCER, J. y S. POLACHEK. "Family Investments in Human Capital: Earnings of Women". Journal of political economy, 82, 76-108, 1974.

Morash, M. y J. Greene. "Evaluating Women on Patrol: A Critic of Contemporary Wisdom”. Evaluation Review, 10, 230-255, 1986.

Morello, Karen. The Invisible Bar: The Women Lawyer in America, 1638 to the Present. New York, Random House, 1949.

Motta, C. y M. RodríGuez. Mujer y Justicia. El caso Argentino. Buenos Aires, Banco Mundial, 2001.

Nieva V. y B. Gutek. Women and Work: A Psychological Perspective. New York, Praeger, 1981.

Olsen, Frances. Feminism and Critical Legal Theory: An American Perspective. The International Journal of the Sociology of Law, 18, 2, 199 . 215, 1995.

—_The Family and the Market: A Study of Ideology and Legal Reform", Harvard Law Review, 96, 1497-1498, 1982-1983.

PADAVIC, I. y B. Reskin. Women and Men at Work. Thousand Oaks, CA, Pine Forge, 2002. 
PARKer, S. y C. Pederzini. "Género y educación en México". Estudios Demográficos y Urbanos, 97-122, 2000.

PAUTASSI, L. "Legislación previsional y equidad de género en América Latina”. Santiago: CEPAL. Serie Mujer y Desarrollo, 42, 2002.

PIERCE, J. Gender Trials: Emotional Lives in Contemporary Law Firms. Berkeley, University of California Press, 1995.

PITCH, Tamar. Un derecho para dos: la construcción jurídica de género, sexo y sexualidad. Madrid, Trotta, 2003.

Reichman, N. y J. Sterling. "Recasting the Brass Ring: Deconstructing and Reconstructing Workplace Opportunities For Women Lawyers". Capital University Law Review, 2, 923-977, 2002.

Reskin, B. "Bringing the Men Back In: Sex Differentiation and the Devaluation of Women’s Work". Gender Ë Society, 2, 58-81, 1998.

RHode, D. (1988); "Perspectives on Professional Women". Stanford Law Review, 40, 1164-1207, 1988.

RODRÍGUEz GÓmEZ, Roberto. "Género y políticas de educación superior en México". La Ventana, 10, 124-159, 1999.

Sachs, A. y J. Wilson. Sexism and the Law. Oxford, Robertson, 1978.

Seron, C. y Ferris, K. "Negotiating Professionalism: the Gendered Social Capital of Flexible Time”. Work and Occupations, 22, 23-47, 1995. Sirianni, C. y A. Welsh. "Through the Prison of Time: Temporal Structures in Post-Modern America". In A. Wolfe (ed.); American at century's end. Berkeley: California University Press, 421-439, 1991. Smart, Carol. Feminism and the Power of Law. London, Routledge, 1989. STURM, S. Second Generation Employment Discrimination: A Structural Approach. Columbia Law Review, 101, 3, 458-568, 2001. 
VillanueVa Flores, Rocío. Sobre Género, Derecho y Discriminación. Lima, PUC Perú/Defensoría del Pueblo, 1999.

West, C. y D. Zimmerman. “Doing Gender”. Gender $\mathcal{G}$ Society, 1, 125-151, 1987.

Wilkins, D. y G. Gulati. "Why Are There So Few Black Lawyers in Corporate Law Firms? An Institutional Analysis". California Law Review, 84, 493-625, 1996. 